\title{
Fusobacterium nucleatum subsp. fusiforme subsp. nov. and Fusobacterium nucleatum subsp. animalis subsp. nov. as Additional Subspecies within Fusobacterium nucleatum
}

\author{
SAHEER E. GHARBIA ${ }^{1 *}$ AND HAROUN N. SHAH ${ }^{2}$ \\ Department of Oral Biology, Faculty of Dentistry, ${ }^{1}$ and Department of Microbiology and Immunology, Faculty of \\ Medicine, ${ }^{2}$ Dalhousie University, Halifax, Nova Scotia B3H 3J5, Canada
}

\begin{abstract}
Using a variety of physiological, biochemical, and molecular systematic analyses, we have shown previously that there are four groups within the species Fusobacterium nucleatum. Two of these groups of strains correspond to the recently proposed taxa $F$. nucleatum subsp. nucleatum and $F$. nucleatum subsp. polymorphum. In this paper we show that the two remaining groups are distinct and formally propose that they should be recognized as $F$. nucleatum subsp. fusiforme (type strain, NCTC 11326) and $F$. nucleatum subsp. animalis (type strain, NCTC 12276). The tests which we used did not allow a full assessment of the status of $F$. nucleatum subsp. vincentii compared with $F$. nucleatum subsp. nucleatum.
\end{abstract}

Fusobacterium nucleatum, the type species of the genus Fusobacterium, has been shown to comprise a heterogeneous collection of strains $(1,3,4)$. Recently, Dzink et al. (2) described three subspecies, $F$. nucleatum subsp. nucleatum, $F$. nucleatum subsp. polymorphum, $F$. nucleatum subsp. vincentii, while almost simultaneously we described four subspecies (5) following a series of detailed systematic analyses $(3-6,8)$. The descriptions of $F$. nucleatum subsp. nucleatum and $F$. nucleatum subsp. polymorphum in two previous studies $(2,5)$ appear to be consistent, and the same type strains and the same names were proposed independently for each subspecies. It is evident from a previous description of " $F$. nucleatum subsp. animalis" (5) that this organism is a distinct taxon within the species $F$. nucleatum. However, the taxonomic position of " $F$. nucleatum subsp. fusiforme" (5) remains unclear. Because of the paucity of tests used for describing $F$. nucleatum subsp. vincentii, it is uncertain whether this subspecies and $F$. nucleatum subsp. fusiforme are synonymous. In this study, we compared strains of $F$. nucleatum subsp. fusiforme with the type strain of $F$. nucleatum subsp. vincentii by using enzymic electrophoretic patterns, which have been shown previously to have significant intraspecies diagnostic value in the genus Fusobacterium. In addition, in this paper we formally propose recognition of $F$. nucleatum subsp. animalis. Our proposals are consistent with our recent rRNA sequence data (9).

\section{MATERIALS AND METHODS}

Bacterial strains. $F$. nucleatum reference strains ATCC $25586^{\mathrm{T}}\left(\mathrm{T}=\right.$ type strain), ATCC $49256^{\mathrm{T}}$, ATCC $10953^{\mathrm{T}}$, NCTC $11326^{\mathrm{T}}$, and NCTC $12276^{\mathrm{T}}$ and 10 clinical isolates resembling strains NCTC $11326^{\mathrm{T}}$ and NCTC $12276^{\mathrm{T}}$ were used in this study.

Growth conditions and identification. All strains were maintained by weekly subculturing on blood agar culture medium containing $5 \%$ (vol/vol) sheep blood (Oxoid) in an

\footnotetext{
* Corresponding author.
}

atmosphere containing $10 \% \mathrm{CO}_{2}$ and $10 \% \mathrm{H}_{2}$ in $\mathrm{N}_{2}$ at $37^{\circ} \mathrm{C}$. Clinical isolates were identified as described previously (4, 7).

Electrophoresis and staining of enzymes. Cell extracts were prepared and electrophoresis was carried out on cellogel support strips as described previously (3). Glutamate dehydrogenase $(\mathrm{GDH})$ and 2-oxoglutarate reductase (OGR) bands were stained as described previously $(3,4)$.

\section{RESULTS AND DISCUSSION}

All of the isolates were identified as members of $F$. nucleatum by their ability to produce indole, their absence of urease activity, and their ability to reduce nitrate. Butyrate and acetate were major acidic end products of metabolism. Six of the clinical isolates which we tested morphologically resembled strain NCTC $11326^{\mathrm{T}}$ (previously designated $F$. nucleatum subsp. fusiforme); the electrophoretic mobilities for GDH and OGR for these strains were 1.8 and $2.0 \mathrm{~cm}$, respectively. The isolates that resembled strain NCTC $12276^{\mathrm{T}}$ (designated $F$. nucleatum subsp. animalis) grew in the presence of $20 \%$ bile and had both GDH and OGR activities (electrophoretic mobilities, 3.2 and $3.8 \mathrm{~cm}$, respectively). Figure 1 shows the GDH electrophoretic mobilities for the five reference strains representing the subspecies examined in this study. $F$. nucleatum subsp. polymorphum strains had a distinct electrophoretic mobility, whereas the GDH of $F$. nucleatum subsp. vincentii coelectrophoresed with $F$. nucleatum subsp. nucleatum GDH. The OGR of $F$. nucleatum subsp. nucleatum and the OGR of the type strain of $F$. nucleatum subsp. vincentii also comigrated (Fig. 1).

In this study we could not distinguish between strains of $F$. nucleatum subsp. nucleatum and the type strain of $F$. nucleatum subsp. vincentii. This could indicate that these two subspecies are synomonous, that an incorrect reference strain of $F$. nucleatum subsp. vincentii has been deposited in a culture collection, or that the tests which we used cannot be used to differentiate these taxa. However, it is clear that $F$. nucleatum subsp. vincentii and $F$. nucleatum subsp. fusiforme are not synonymous as is sometimes stated (12). 


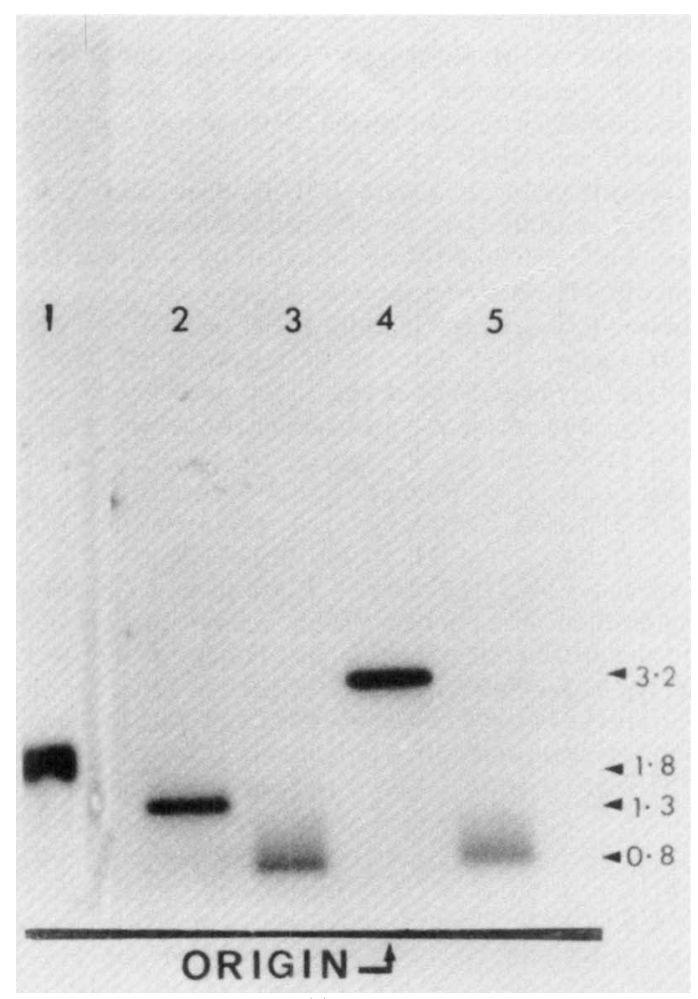

FIG. 1. GDH electrophoretic mobilities of $F$. nucleatum subsp. fusiforme (lane 1), $F$. nucleatum subsp. polymorphum (lane 2), $F$. nucleatum subsp. nucleatum (lane 3 ), $F$. nucleatum subsp. animalis (lane 4), and $F$. nucleatum subsp. vincentii (lane 5).

The strains assigned to $F$. nucleatum subsp. animalis had the fastest-migrating enzymes on electropherograms and formed a distinct cluster that was readily recognizable from the other subspecies. When both GDH and OGR electrophoretic patterns were used as a basis for comparison, the strains assigned to $F$. nucleatum subsp. fusiforme (including strain NCTC $11326^{\mathrm{T}}$ ) were clearly different from $F$. nucleatum subsp. vincentii ATCC 49256; thus, separate subspecies status for $F$. nucleatum subsp. fusiforme was justified.

In previous studies we reported the absence of any clear physiological tests that can be used to differentiate among the subspecies of $F$. nucleatum. Furthermore, the levels of DNA-DNA homology when experiments are carried out under optimal hybridization conditions are high (4). Under stringent hybridization conditions, however, four centers of variations are clearly evident; these correlate with the groups formed on the basis of the GDH and OGR electrophoretic patterns (4). These groups have also been substantiated by rRNA gene restriction patterns, using a plasmid (pK3535) to probe chromosomal DNA digests of the subspecies (8). More recently, we found high levels of rRNA sequence similarity (ca. 97.3 to $98.4 \%$ ) that are consistent with a single species. However, an unrooted phylogenetic tree based on $K_{\text {nuc }}$ (envolutionary distance) values clearly delineated the four taxa. Therefore, we formally propose that $F$. nucleatum subsp. fusiforme and $F$. nucleatum subsp. animalis, which until now have been unclassified, should be recognized as new subspecies of $F$. nucleatum. Descriptions of these two taxa are given below.

Description of Fusobacterium nucleatum subsp. fusiforme subsp. nov., nom. rev., comb. nov. Fusobacterium nuclea- tum subsp. fusiforme (fu.si.for'me. L.n. fusus, a spindle; L.n. forma, shape; M.L. adj. fusiforme, spindle shaped). Gram negative. Anaerobic. Cells are rod shaped and nonmotile, do not form filaments, and are 8 to $16 \mu \mathrm{m}$ long by 0.5 $\mu \mathrm{m}$ in diameter. Colonies on blood agar after $24 \mathrm{~h}$ are granular with very irregular edges, 10 to $13 \mu \mathrm{m}$ in diameter, and low cone shaped, and pitting is frequently observed if colonies are dislodged. Trypticase, protease peptone, and Bacto Casitone (Difco) markedly enhance growth in liquid medium. Few amino acids (mainly glutamate, histidine, cysteine, and serine) are utilized. The terminal $\mathrm{pH}$ in a glucose medium is higher than 7.0. The electrophoretic mobilities of GDH and OGR are 1.8 and $2 \mathrm{~cm}$, respectively. Moderate levels of $\mathrm{H}_{2} \mathrm{~S}$ are produced in liquid medium. All strains possess the constitutive enzymes leucine aminopeptidase and gamma-glutamyl aminopeptidase. Serine-valine aminopeptidase and proline aminopeptidase are not produced. Glycosidases are absent.

The cell wall peptidoglycan contains meso-lanthionine as its diamino acid. Possesses straight-chain, monounsaturated, and hydroxylated long-chain fatty acids but not methyl-branched acids. Tetradecanoic acid $\left(\mathrm{C}_{14: 0} ; \mathrm{ca} .24\right.$ to $30 \%)$ and hexadecanoic acid $\left(\mathrm{C}_{16: 0} ; 20\right.$ to $\left.26 \%\right)$ are the prodominant nonhydroxylated acids. The major hydroxylated fatty acids are 3-hydroxytetradecanoic acid $(3 \mathrm{OH}-$ $\mathrm{C}_{14: 0} ;$ ca. 12 to $\left.18 \%\right)$ and 3-hydroxyhexadecanoic acid (3OH$\mathrm{C}_{16: 0}$; ca. 4 to $7 \%$ ). The DNA base composition is 26 to 28 mol\% $\mathrm{G}+\mathrm{C}$, as determined by the thermal denaturation method. The type strain is strain NCTC 11326.

Description of Fusobacterium nucleatum subsp. animalis subsp. nov. Fusobacterium nucleatum subsp. animalis (a.ni.ma'lis. L. n. animal, animal; animalis, of an animal). Gram negative. Anaerobic. Cells are nonmotile rods that occasionally form filaments which are up to $25 \mu \mathrm{m}$ long. Colonies on blood agar are round, smooth, glistening, convex, translucent white, and 1.0 to $2.0 \mu \mathrm{m}$ in diameter. Growth is markedly affected by the addition of Trypticase, protease peptone, and Bacto Casitone (Difco). Few amino acids (mainly histidine, arginine, cysteine, and, to a lesser extent, glutamate) are utilized. The terminal $\mathrm{pH}$ in a glucose liquid medium ranges between 6.8 and 7.0. Both GDH and OGR are present and exhibit fast electrophoretic mobilities (3.2 and $3.8 \mathrm{~cm}$, respectively). Low levels of $\mathrm{H}_{2} \mathrm{~S}$ are produced, and growth is not affected by the addition of $20 \%$ bile. Strains are morphologically similar to $F$. nucleatum subsp. polymorphum, but the level of DNA-DNA homology between these two subspecies ranges between 60 and $67 \%$. All strains possess the constitutive enzymes leucine aminopeptidase and gamma-glutamyl aminopeptidase. Serinevaline aminopeptidase and proline aminopeptidase are not produced. Glycosidases are absent.

The cell wall peptidoglycan contains meso-lanthionine as its diamino acid. Possesses straight-chain monounsaturated, and hydroxylated long-chain fatty acids but not methylbranched acids. Tetradecanoic acid $\left(\mathrm{C}_{14: 0}\right.$; ca. 24 to $\left.30 \%\right)$ and hexadecanoic acid $\left(\mathrm{C}_{16: 0} ; 20\right.$ to $\left.26 \%\right)$ are the major nonhydroxylated acids. The major hydroxylated fatty acids are 3-hydroxytetradecanoic acid $\left(3 \mathrm{OH}-\mathrm{C}_{14: 0}\right.$; ca. 12 to $\left.18 \%\right)$ and 3-hydroxyhexadecanoic acid $\left(3 \mathrm{OH}-\mathrm{C}_{16: 0}\right.$; ca. 4 to $\left.7 \%\right)$. The DNA base composition is 26 to $28 \mathrm{~mol} \% \mathrm{G}+\mathrm{C}$, as determined by the thermal denaturation method. The type strain is strain NCTC 12276.

\section{ACKNOWLEDGMENT}

This work was supported by Development Grant number DG-411 from the Medical Research Council of Canada. 


\section{REFERENCES}

1. Calhoon, D. A., W. R. Mayberry, and J. Slots. 1983. Cellular fatty acid and soluble protein profiles of oral fusobacteria. J. Dent. Res. 62:1181-1185.

2. Dzink, J. L., M. T. Sheenan, and S. S. Socransky. 1990. Proposal of three subspecies of Fusobacterium nucleatum Knorr 1922: Fusobacterium nucleatum subsp. nucleatum subsp. nov., comb. nov.; Fusobacterium nucleatum subsp. polymorphum subsp. nov., nom. rev., comb. nov.; and Fusobacterium nucleatum subsp. vincentii subsp. nov., nom. rev., comb. nov. Int. J. Syst. Bacteriol. 40:74-78.

3. Gharbia, S. E., and H. N. Shah. 1988. Characteristics of glutamate dehydrogenase, a new diagnostic marker for the genus Fusobacterium. J. Gen. Microbiol. 134:327-332.

4. Gharbia, S. E., and H. N. Shah. 1989. Glutamate dehydrogenase and 2-oxoglutarate reductase electrophoretic patterns and deoxyribonucleic acid-deoxyribonucleic acid hybridization among human, oral isolates of Fusobacterium nucleatum. Int. J. Syst. Bacteriol. 39:467-470.

5. Gharbia, S. E., and H. N. Shah. 1990. Heterogeneity within Fusobacterium nucleatum, proposal of four subspecies. Lett. Appl. Bacteriol. 10:105-108.

6. Gharbia, S. E., and H. N. Shah. 1990. Identification of Fusobacterium species by the electrophoretic migration of glutamate dehydrogenase and 2-oxoglutarate reductase. J. Med. Micro- biol. 33:183-188.

7. Gharbia, S. E., H. N. Shah, P. A. Lawson, and M. Haapasalo. 1990. The distribution and frequency of Fusobacterium nucleatum subspecies in the human oral cavity. Oral Microbiol. Immunol. 5:324-327.

8. Lawson, P. A., S. E. Gharbia, H. N. Shah, and D. R. Clark. 1989. Recognition of Fusobacterium nucleatum subgroups Fn-1, Fn-2 and Fn-3 by ribosomal RNA gene restriction patterns. FEMS Microbiol. Lett. 65:41-46.

9. Lawson, P. A., S. E. Gharbia, H. N. Shah, D. R. Clark, and M. D. Collins. 1991. Intrageneric relationships of members of the genus Fusobacterium as determined by reverse transcriptase sequencing of small-subunit RNA. Int. J. Syst. Bacteriol. 41:347-354.

10. Magee, J. T., J. M. Hindmarch, K. W. Bennett, B. I. Duerden, and R. E. Aries. 1989. A pyrolysis mass spectrometry study of fusobacteria. J. Med. Microbiol. 28:227-236.

11. Vasstrand, E. N., H. B. Jansen, T. Miron, and T. Hofstad. 1982. Composition of peptidoglycans in Bacteroidaceae: determination and distribution of lanthionine. Infect. Immun. 36:114-122.

12. Xie, H., R. J. Gibbons, and D. I. Hay. 1991. Adhesive properties of strains of Fusobacterium nucleatum of the subspecies nucleatum, vincentii and polymorphum. Oral Microbiol. Immunol. 6:257-263. 